\title{
基于柔性并联连续体的灵巧操作手的设计及分析
}

\author{
汪培义 郭 盛 王向阳 宋马军 林华杰
}

(北京交通大学机械与电子控制工程学院 北京 100044)

\begin{abstract}
摘要: 提出了一种新型 4-DOF 柔性并联连续体机构, 将该机构与末端绳驱动的抓手相结合, 设计完成具有灵活抓取性能的操 作手。将该柔性机构通过柔性支链等效的方式等效为传统刚性连杆的并联机构, 并且运用螺旋理论对该机构进行自由度分析; 采用经典的 Cosserat Rod 模型对机构进行运动学建模; 通过对比分析三种并联连续体机构的可操作度, 4-DOF 并联连续体机 构由于具有一个额外的扭转自由度, 使得操作手能够更加灵活地抓取物体, 在此基础上给出了一组操作手抓取的仿真实例。 最后通过试验验证机构自由度以及运动学模型的数值解, 并且实现操作手灵活抓取的性能。
\end{abstract}

关键词: 并联连续体机构; Cosserat Rod 模型; 操作手; 灵活抓取; 运动可操作度 中图分类号: TG156

\section{Design and Analysis of a Dexterous Gripper Based on Flexible Parallel Continuum Manipulator}

\author{
WANG Peiyi GUO Sheng WANG Xiangyang SONG Majun LIN Huajie
}

(School of Mechanical, Electronic and Control Engineering, Beijing Jiaotong University, Beijing 100044)

\begin{abstract}
A new four-degree-of-freedom parallel continuum manipulator is presented, which was combined with a cable-driven gripper to improve the performance of dexterous grasp. This manipulator is approximately equivalent to the traditional rigid link parallel mechanism by complaint branch equivalent method, and the degree of freedom analysis of the manipulator is performed by using the screw theory. The classic Cosserat Rod model is applied to model the kinematics of the continuum manipulator. By comparative analysis among three types of parallel continuum manipulator, the proposed manipulator can provide an additional torsional movement which make the gripper grasp the object more flexibly. Based on this, a simulation example of dexterous grasp is given. Finally, experiments verify the degree of freedom and kinematics of the manipulator and the performance of flexible grasping of the gripper.
\end{abstract}

Key words: parallel continuum manipulator; Cosserat Rod model; gripper; flexible grasp; kinematic manipulability

\section{0 前言}

连续体机器人整体形状连续变化, 结构单元多 样, 应用范围十分广泛, 从手术机器人的远程操作 到工业机器人的灵活抓取 ${ }^{[1]}$ 。其中并联连续体机构 由边界约束平台以及并联排列的柔性支链组成 ${ }^{[2]}$, 由于结构的固有柔性和低质量, 基于该机构设计而 成的操作手在与人交互时更加的安全, 并且在限定 空间中提供准确的多自由度运动, 增强环境适应性,

* 国家自然科学基金(51875033)和北京市自然科学基金(L172021)资助项 目。20200221 收到初稿, 20200506 收到修改稿
同时可以利用自身柔性抵抗外界环境的冲击, 避免 损坏 ${ }^{[3]}$ 。与传统的并联刚性连杆操作手相比, 在结 构、轻量化和柔顺性方面具有明显优势, 与串联连 续体操作手相比, 它具有更高的载荷能力、准确性 和可重复性。

SIMAAN 等 ${ }^{[4]}$ 设计了 3-DOF 多杆连续体机构, 并将其应用到远程操作灵巧手术工具的腕关节设计 中, 通过在末端增加可拆卸的抓手, 实现操作手的 灵巧操作。徐凯等 ${ }^{[5]}$ 提出了多杆连续体机构的广义 构型, 通过这种广义构型的组合和连接, 能够实现 多领域的应用, 如应用在内窥镜和腹腔镜手术机器 人 $^{[6-7]}$, 工业深腔机械臂 ${ }^{[8]}$ 、肩部康复外骨骼 ${ }^{[9-10]}$ 、 假肢手 ${ }^{[11-12]}$ 以及基于连续体关节的并联机构 ${ }^{[13]}$ 的设 
计当中。此外, 德国 Festo 公司研制的 3-DOF 仿生 三指操作手 ${ }^{[14]}$, 通过使用四根柔性驱动杆穿过一个 中间约束平台并联连接, 能够为末端操作手提供大 范围的工作空间。连续体机构的发展 ${ }^{[1]}$ 和传统并联 机构的发展为并联连续体机构的提出提供了一定的 基础, 其中 BRYSON 等 ${ }^{[2]}$ 首次提出了与传统的刚性 连杆 Stewart-Gough 并联机构相似的 6-DOF 并联连 续体机构, 并利用经典的 Cosserat Rod 模型对该机 构的运动学和动力学 ${ }^{[15]}$ 进行准确分析。OREKHOV 等 ${ }^{[16]}$ 将 6-DOF 并联连续体机构与绳驱动的抓手相 结合应用于手术内镜检查中, 将这种小型化多自由 度的操作手应用于空间限定的手术操作中。

YOUNG 等设计了 6-DOF 并联连续体机构 ${ }^{[17]}$, 并将 其应用于指尖可穿戴设备中, 用来传递指尖的触觉 线索 ${ }^{[18]}$ 。林华杰等 ${ }^{[19]}$ 设计了 $3-\mathrm{DOF}$ 并联连续体机 构, 采用 D-H 法对机构进行运动学分析, 最后通过 仿真验证了运动模型。WU 等 ${ }^{[20]}$ 设计了多约束平台 并联连续体机构, 并且提供了基于末端数据的标定 方法, 通过试验确定了该方法具有较高的精度。

从以上研究成果来看, 多杆连续体机构的研究 和分析都较为广泛, 并且应用范围较大, 以并联机 构为原型的并联连续体机构的设计和应用较少, 由 于并联连续体机构具有较多的自由度, 能够为末端 平台提供更加灵活的运动, 此外由于该机构动静平 台之间通过柔性连杆连接, 它的柔顺性、轻量化以 及人机交互的安全性更高。

本文设计提出了一种新型的 4-DOF 并联连续 体机构; 采用柔性支链等效和螺旋理论的方法, 对 提出的机构进行了自由度分析; 应用经典的 Cosserat $\operatorname{Rod}$ 模型分析该机构的运动学, 并给出数值解; 通 过将该机构与末端绳驱动的抓手相结合, 设计完成 灵巧操作手的实验样机; 最后通过试验验证了理论 模型的正确性, 并且实现了操作手的灵活抓取。

\section{1 柔性并联连续体的自由度分析}

\section{1 机构描述}

图 1 所示为 4 支链并联连续体机构, 该机构由 动静平台和 4 条柔性支链构成。支链 1, 2, 3 的末端 与动平台固定连接, 支链 4 通过螺旋副( $\mathrm{H}$ 副)与动 平台连接, $A_{e} 、 B_{e} 、 C_{e}$ 和 $e$ 点分别为各支链与动平 台的连接点。各支链与静平台通过移动副 $(\mathrm{P}$ 副)的形 式连接, 分别交于 $A 、 B 、 C$ 和 $O$ 点, 支链相对于 静平台能够沿着轴向方向移动, 因此动静平台之间 每条支链的长度是可变的。

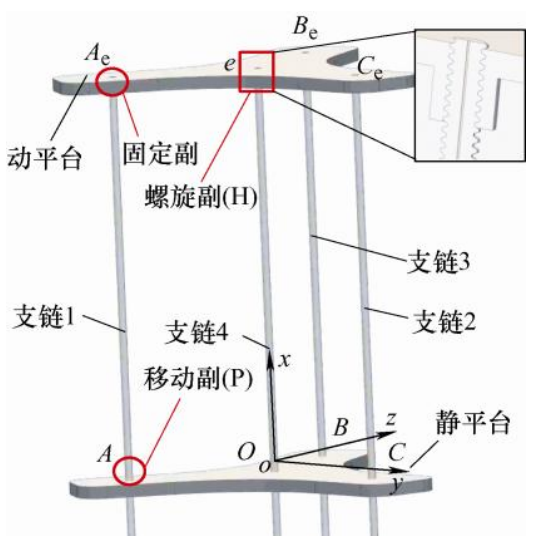

图 1 新型 4 支链并联连续体机构

\section{2 自由度计算}

图 2 所示为支链 1 等效运动示意图, 超弹性镍 钛合金杆的扭转模量较大, 因此忽略柔性支链的扭 转变形, 柔性支链在静平台 $A$ 点处绕平面 $a$ 内任意 轴线 $l_{0}$ 旋转, 在动平台 $A_{e}$ 点处绕平面 $b$ 内任意轴线 $l_{e}$ 旋转, 在整个机构运动过程中, 点 $A$ 和 $A_{e}$ 相对于 平台不会发生变化, 轴线 $l_{0}$ 和 $l_{e}$ 始终在平面 $a, b$ 内。 由于 $A$ 点处支链以移动副的形式与静平台连接, 所 以 $A$ 和 $A_{e}$ 点之间支链 1 的长度是可变的。

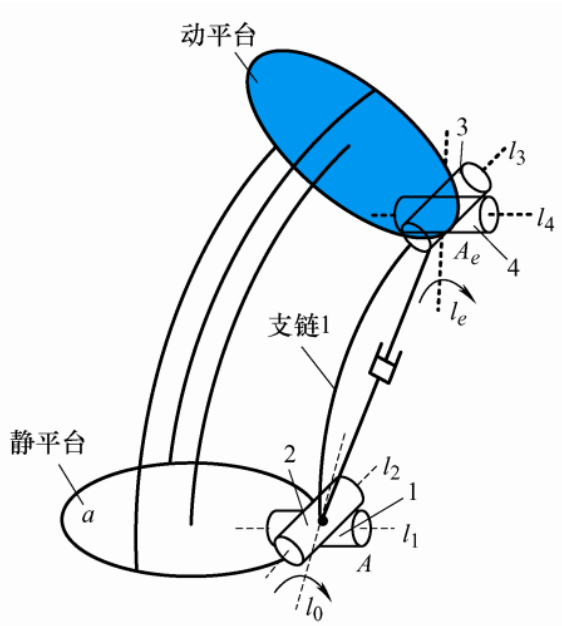

图 2 支链 1 等效运动示意图

假设在点 $A$ 和 $A_{e}$ 处分别存在两个转动副 1,2 和 3,4 , 它们的轴线 $l_{1}, l_{2}$ 和 $l_{3}, l_{4}$ 在各自的平面内汇交于 一点, 即线性无关, 则轴线 $l_{0}$ 和 $l_{e}$ 分别可由 $l_{1}, l_{2}$ 和 $l_{3}, l_{4}$ 线性表示

$$
\begin{aligned}
& \boldsymbol{l}_{\mathbf{0}}=c_{1} \boldsymbol{l}_{1}+c_{2} \boldsymbol{l}_{2} \\
& \boldsymbol{l}_{e}=c_{3} \boldsymbol{l}_{3}+c_{4} \boldsymbol{l}_{4}
\end{aligned}
$$

因此支链 1 绕着轴线 $l_{0}$ 和 $l_{e}$ 的运动可以等效为 绕着 $l_{1}, l_{2}$ 和 $l_{3}, l_{4}$ 的运动, 在 $A$ 和 $A_{e}$ 点处选取轴线互 相垂直的 $U$ 副等效运动。柔性支链长度的变化可以 等效为 $\mathrm{P}$ 副的运动。机构在初始位置时, 柔性支链 
不会产生扭转, 轴线 $l_{2}, l_{3}$ 和 $l_{1}$ 和 $l_{4}$ 分别相互平行。

根据上述分析, 将图 1 的 4 支链柔性并联连续 体等效为图 3 的 3-UPU/UPUH 并联机构, 同一支链 中 $U$ 副与动静平台相连的为第一转动副, 与移动副 相连的为第二转动副 ${ }^{[21]}$, 两个 $U$ 副中第一转动副和 第二转动副分别相互平行。

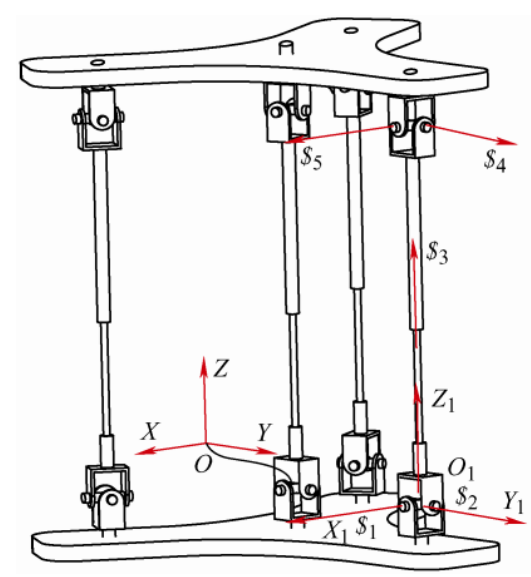

图 3 等效的 3-UPU/UPUH 并联机构

建立图 3 所示的支链坐标系, 原点 $O_{1}$ 取在 $\mathrm{U}$ 副的转动中心点, $X_{1}$ 轴方向平行于支链 $\mathrm{U}$ 副第一转 动副, $Y_{1}$ 轴方向平行于支链 $\mathrm{U}$ 副第二转动副, $Z_{1}$ 轴 按右手定则。可以得到支链 $i(i=1,2,3)$ 的运动螺旋 系为

$$
\begin{aligned}
& \$_{i 1}=\left(\begin{array}{llllll}
1 & 0 & 0 ; & 0 & 0 & 0
\end{array}\right) \\
& \$_{i 2}=\left(\begin{array}{llllll}
0 & 1 & 0 ; & 0 & 0 & 0
\end{array}\right) \\
& \$_{i 3}=\left(\begin{array}{llllll}
0 & 0 & 0 ; & 0 & 0 & 1
\end{array}\right) \\
& \$_{i 4}=\left(\begin{array}{llllll}
0 & 1 & 0 ; & 0 & 0 & b_{4}
\end{array}\right) \\
& \$_{i 5}=\left(\begin{array}{llllll}
1 & 0 & 0 ; & 0 & 0 & b_{5}
\end{array}\right)
\end{aligned}
$$

求得反螺旋为

$$
\begin{aligned}
\boldsymbol{\$}_{i 1}^{r} & =\left(\begin{array}{llllll}
0 & 1 & 0 ; & 0 & 0 & 0
\end{array}\right) \\
\boldsymbol{\$}_{i 2}^{r} & =\left(\begin{array}{llllll}
1 & 0 & 0 ; & 0 & 0 & 0
\end{array}\right) \\
\boldsymbol{\phi}_{i 3}^{r} & =\left(\begin{array}{llllll}
0 & 0 & 0 ; & 0 & 0 & 1
\end{array}\right)
\end{aligned}
$$

以同样的方法求得支链 4 的反螺旋

$$
\begin{aligned}
& \boldsymbol{\$}_{41}^{r}=\left(\begin{array}{llllll}
0 & 1 & 0 ; & 0 & 0 & 0
\end{array}\right) \\
& \$_{42}^{r}=\left(\begin{array}{llllll}
1 & 0 & 0 ; & 0 & 0 & 0
\end{array}\right)
\end{aligned}
$$

上述约束螺旋约束动平台在空间中 $X-Y$ 平面内 的移动, 因此 4 支链并联连续体机构具有 4 个自由 度, 分别能够实现沿着 $Z$ 轴的移动、绕着 $Z$ 轴的扭 转和绕 $X, Y$ 轴的弯曲。

\section{2 理论建模分析}

利用经典 Cosserat Rod 模型对并联连续体机构
进行建模分析, 该方法能够有效解决外界负载、曲 率连续变化的形状以及非直线约束路径对连续体机 构的影响 ${ }^{[22-23] 。}$

\subsection{Cosserat Rod 模型}

对弹性杆进行建模分析, 图 4 所示为弹性杆坐 标系定义以及形变。建立全局坐标系 $o-x y z$, 杆上每 一个片段的坐标位置表示成关于弧长的矢量 $\boldsymbol{r}(s) \in$ $\mathbf{R}^{3}$, 其中 $\boldsymbol{r}(0)$ 代表着棒的初始位置, $\boldsymbol{r}(s)$ 代表距离起 始点 $s$ 的位置, 在 $\boldsymbol{r}(s)$ 处建立局部坐标系 $o-x_{s} y_{s} z_{s}$, 其 $z_{s}$ 轴沿着杆的切向方向, 旋转矩阵 $\boldsymbol{R}(s) \in S O(3)$ 表示在点 $\boldsymbol{r}(s)$ 处杆的姿态。带有 $l$ 上标的矢量表示在 局部坐标系中的表达。

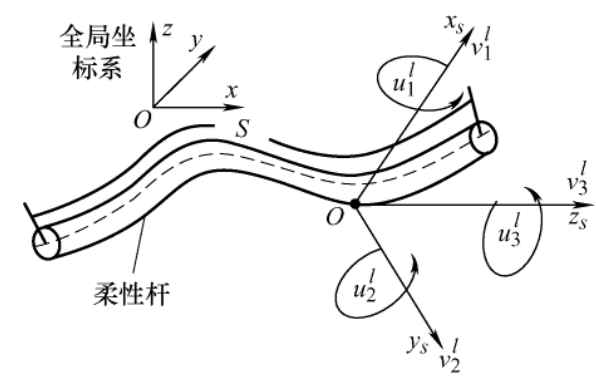

图 4 弹性杆坐标系定义以及形变

单一柔性杆的 Cosserat Rod 微分方程组为

$$
\begin{aligned}
& \boldsymbol{u}^{l}=\boldsymbol{K}_{\mathrm{t}}^{-1} \boldsymbol{R}^{\mathrm{T}} \boldsymbol{m}+\boldsymbol{u}^{l^{*}} \\
& \boldsymbol{v}^{l}=\boldsymbol{K}_{\mathrm{s}}^{-1} \boldsymbol{R}^{\mathrm{T}} \boldsymbol{n}+\boldsymbol{v}^{l^{*}} \\
& \boldsymbol{r}^{\prime}=\boldsymbol{R} \boldsymbol{v}^{l} \\
& \boldsymbol{R}^{\prime}=\boldsymbol{R} \hat{\boldsymbol{u}}^{l} \\
& \boldsymbol{n}^{\prime}=-\boldsymbol{f} \\
& \boldsymbol{m}^{\prime}=-\boldsymbol{r}^{\prime} \times \boldsymbol{n}
\end{aligned}
$$

$$
\boldsymbol{K}_{\mathrm{s}}=\left[\begin{array}{ccc}
G A_{\mathrm{T}} & 0 & 0 \\
0 & G A_{\mathrm{T}} & 0 \\
0 & 0 & E A_{\mathrm{T}}
\end{array}\right] \boldsymbol{K}_{\mathrm{t}}=\left[\begin{array}{ccc}
E I_{x y} & 0 & 0 \\
0 & E I_{x y} & 0 \\
0 & 0 & G J
\end{array}\right]
$$

微分方程组式(1)描述了柔性杆的形状、内力和 内力矩, 状态变量 $\boldsymbol{r}, \boldsymbol{R}, \boldsymbol{n}$ 和 $\boldsymbol{m}$ 是关于弧长 $s$ 的函数。 其中 $\boldsymbol{n}$ 和 $\boldsymbol{m}$ 在世界坐标系中表示, $\boldsymbol{v}^{l *}(s)$ 和 $\boldsymbol{u}^{l *}(s)$ 为 杆长在无应力参考作用下的运动学变量, 对于初始 柔性杆为长直杆时 $\boldsymbol{v}^{l *}(0)=\left[\begin{array}{lll}0 & 0 & 1\end{array}\right]^{\mathrm{T}}$ 和 $\boldsymbol{u}^{l^{*}}(0)=\left[\begin{array}{lll}0 & 0 & 0\end{array}\right]^{\mathrm{T}}$ 。 $\boldsymbol{K}_{\mathrm{s}}$ 和 $\boldsymbol{K}_{\mathrm{t}}$ 为横截面杆的刚度矩阵, $A_{T}$ 为横截面积, $E$ 为弹性模量, $G$ 为切变模量, $I_{x y}$ 为关于 $x y$ 轴的惯性 矩, $J$ 为关于 $z$ 轴的惯性矩。 $\hat{\boldsymbol{u}}^{l}$ 为三维矢量 $\boldsymbol{u}^{l}$ 的反 对称矩阵, 可表达为

$$
\hat{\boldsymbol{u}}=\left[\begin{array}{ccc}
0 & -u_{3} & u_{2} \\
u_{3} & 0 & -u_{1} \\
-u_{2} & u_{1} & 0
\end{array}\right]
$$




\section{2 柔性杆末端边界条件}

图 5 给出了 4-DOF 并联连续体边界条件简图, 动静平台通过并联连接的弹性杆连接, 图中 $A, B, C$, $A_{e}, B_{e}, C_{e}, O$ 和 $e$ 点为各柔性连杆与动静平台之间的 连接点。支链 4 采用 $\mathrm{H}$ 副连接柔性杆和末端平台。 由于不考虑加速度的影响, 机构在不同位型之间的 运动可以看作是缓慢匀速变化, 整个机构在不同位 型时处于静态平衡状态。

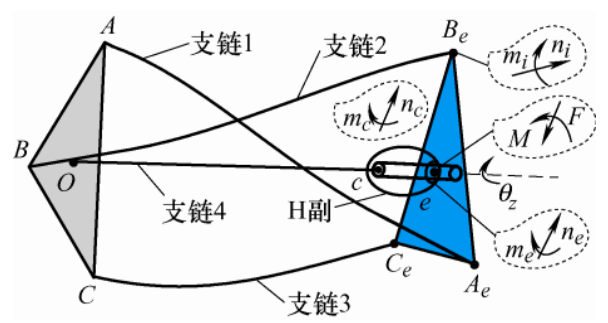

图 5 4-DOF 并联连续体边界条件简图

静态状态下, 末端平台满足力和力矩平衡条件

$$
\begin{aligned}
& \sum_{i=1}^{n}\left[\boldsymbol{n}_{i}\left(L_{i}\right)\right]+\boldsymbol{n}_{\boldsymbol{e}}-\boldsymbol{F}=0 \\
& \sum_{i=1}^{n}\left[\boldsymbol{p}_{i}\left(L_{i}\right) \times \boldsymbol{n}_{i}\left(L_{i}\right)+\boldsymbol{m}_{i}\left(L_{i}\right)\right]+ \\
& \boldsymbol{p}_{e} \times \boldsymbol{n}_{e}+\boldsymbol{m}_{e}-\boldsymbol{p}_{e} \times \boldsymbol{F}-\boldsymbol{M}=0
\end{aligned}
$$

$$
i=1,2,3
$$

式中, $\boldsymbol{F}$ 和 $\boldsymbol{M}$ 为作用于末端平台中心点 $\boldsymbol{p}_{\boldsymbol{e}}$ 处的外部 力和力矩。 $\boldsymbol{p}_{i}\left(L_{i}\right)$ 为长为 $L$ 的柔性杆末端相对于世界 坐标系的位置, $\boldsymbol{n}_{i}\left(L_{i}\right)$ 和 $\boldsymbol{m}_{i}\left(L_{i}\right)$ 为柔性杆末端产生的 力和力矩, $\boldsymbol{n}_{e}$ 和 $\boldsymbol{m}_{e}$ 为支链 4 通过螺旋副对末端平台 的作用力和力矩。

令支链 4 末端轴向力 $\boldsymbol{F}_{0}=\boldsymbol{e}_{3}^{\mathrm{T}} \boldsymbol{R}_{c}^{\mathrm{T}}\left(L_{c}\right) \boldsymbol{n}_{c}\left(L_{c}\right)$, 则 在动平台产生的力和力矩

$$
\begin{aligned}
& \boldsymbol{n}_{e}=\boldsymbol{n}_{c}\left(L_{c}\right) \\
& \boldsymbol{m}_{e}=\boldsymbol{R}_{c}\left(L_{c}\right)\left(\boldsymbol{e}_{3}^{\mathrm{T}} \boldsymbol{R}_{c}^{\mathrm{T}}\left(L_{c}\right) \boldsymbol{m}_{c}\left(L_{c}\right)+f\left(\boldsymbol{F}_{0}\right)\right) \\
& f\left(\boldsymbol{F}_{0}\right)=\boldsymbol{F}_{0} \frac{d_{2}}{2} \tan \left(\phi+\varphi_{v}\right)
\end{aligned}
$$

式中, $f\left(\boldsymbol{F}_{0}\right)$ 为映射函数, 表示驱动杆末端轴向力 与螺旋机构在动平台产生的力矩之间的关系, $d_{2}$ 为 螺旋副中径, $\varphi_{v}$ 为螺旋副的当量摩擦角, $\varphi$ 为螺纹 升角。

所有柔性支链在末端平台需要满足位置和姿态 约束条件

$$
\begin{aligned}
& \boldsymbol{p}_{e}+\boldsymbol{R}_{e} \mathbf{r}_{i}-\boldsymbol{p}_{i}=0 \quad i=1,2,3 \\
& {\left[\log \left(\boldsymbol{R}_{e}^{\mathrm{T}} \boldsymbol{R}_{i}\left(L_{i}\right)\right)\right]^{\vee}=0 \quad i=1,2,3}
\end{aligned}
$$

$$
\begin{aligned}
\boldsymbol{R}_{e} & =\boldsymbol{R}_{c}\left(L_{c}\right) \boldsymbol{R}_{z}^{l}(\theta) \\
\boldsymbol{p}_{e} & =\boldsymbol{p}_{c}+\boldsymbol{R}_{c}\left(L_{c}\right) \boldsymbol{l}_{e}
\end{aligned}
$$

式中, $\boldsymbol{R}_{z}^{l}\left(\theta_{z}\right)$ 为末端平台在螺旋副的作用下旋转角 度的姿态矩阵(在柔性杆末端坐标系中表达)。

\section{3 柔性杆初值状态条件}

由于该机构各支链通过移动副的形式与静平台 连接, 因此采用直线驱动器驱动柔性杆, 从而实现 各个支链与静平台的相对移动。因此在弧长 $s=0$ 时, 柔性杆的位置和姿态是已知的, 而基座施加的约束 和约束力矩是未知的。

\section{4 数值解法}

图 6 所示为采用打靶法求解边界值的过程。利 用该方法求解 4-DOF 并联连续体机构的运动学, 表 1 给出了求解过程中的未知初值 $\boldsymbol{u}$ 和约束条件 $\boldsymbol{r}$ 。求 解过程如下: 首先给定表 1 中 24 个未知初值, 并将 其写入矩阵 $\boldsymbol{u}$ 中, 其次将每个支链的约束条件写入 矩阵 $\boldsymbol{r}$ 中, 接着在每一组 $\boldsymbol{u}$ 的情况下, 利用四阶龙 格——库塔对 ODE 方程组进行数值积分求解, 从而 评估残差矢量 $\boldsymbol{r}$, 最后利用打靶法更新未知初始值, 直到残差满足一定的误差限或者迭代到最大值时才 停止。

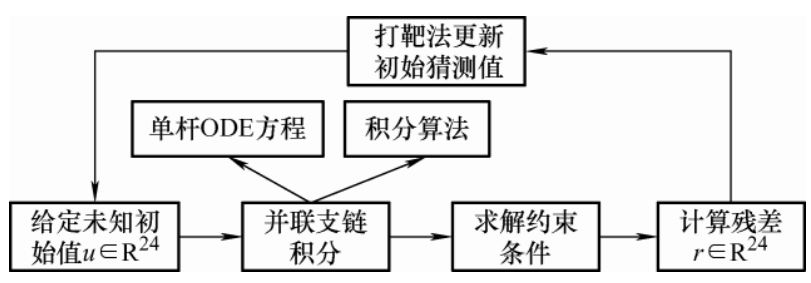

图 6 打靶法求解边界值模型

表 1 正运动学末知初值和约束条件

\begin{tabular}{ccc}
\hline 支链 & 未知初值 $u_{i}$ & 约束条件 $r_{i}$ \\
\hline$i=1,2,3$ & & 约束式(2)、(4)、(5) \\
$i=4$ & {$\left[\boldsymbol{n}_{i}(0) ; \boldsymbol{m}_{i}(0)\right]_{6 \times 1}$} & 约束式(2) $\sim(6)$ \\
\hline
\end{tabular}

\section{3 操作手运动抓取分析}

\section{1 运动学数值解}

给定每个柔性支链的弧长, 确定驱动端的驱动 量, 对各支链的驱动进行不同配置能够实现末端平 台不同的运动, 从而求解出末端平台的位置和姿态。 表 2 给出了 4-DOF 并联连续体 9 组正运动学数值算 例, 考虑动平台和抓手的重力对整个并联连续体机 构的影响, 因此在外力 $F=1.373 \mathrm{~N}$ 的作用下求解运 动学数值解。图 7 给出了表 2 中的三种正运动学驱 动位型。 
表 2 4-DOF 并联连续体正运动学数值算例(外力 $F=1.373 \mathrm{~N}$ )

\begin{tabular}{|c|c|c|c|c|}
\hline 驱动 & $\begin{array}{c}\text { 支链 } 1 \text { 长度 } \\
L_{1} / \mathrm{mm}\end{array}$ & $\begin{array}{c}\text { 支链 } 2 \text { 长度 } \\
L_{2} / \mathrm{mm}\end{array}$ & $\begin{array}{c}\text { 支链 } 3 \text { 长度 } \\
L_{3} / \mathrm{mm}\end{array}$ & $\begin{array}{r}\text { 支链 } \\
L_{4}\end{array}$ \\
\hline 1 & 257.0 & 257.0 & 257.0 & 21 \\
\hline 2 & 257.0 & 257.0 & 257.0 & 2 \\
\hline 3 & 257.0 & 257.0 & 257.0 & \\
\hline 4 & 272.0 & 272.0 & 272.0 & 2 \\
\hline 5 & 272.0 & 272.0 & 272.0 & 2 \\
\hline 6 & 272.0 & 272.0 & 272.0 & 22 \\
\hline 7 & 272.0 & 272.0 & 272.0 & 2 \\
\hline 8 & 272.0 & 272.0 & 272.0 & 21 \\
\hline 9 & 272.0 & 272.0 & 272.0 & 2 \\
\hline
\end{tabular}

图 7 4-DOF 并联连续体驱动位型

\section{2工作空间}

柔性并联连续体机构的工作空间决定了末端操 作手的工作范围。在各支链长度 $L_{1} 、 L_{2} 、 L_{3} 、 L_{4} \in$ $(0.15 \mathrm{~m}, 0.25 \mathrm{~m})$, 通过扫描给定不同的驱动配置, 得到末端操作手的工作空间。图 8 所示为该操作手 的工作空间，图 $8 \mathrm{~b} 、 8 \mathrm{c}$ 为工作空间 $y-z$ 平面视图和 $x-y$ 平面视图。由于连续体机构是对称的, 因此该机 构的工作空间呈对称特性。

\section{3 可操作度}

针对机器人和操作手的灵活性能, 可操作度可 以反映操作手在某一给定位形附近的操作能力 ${ }^{[24]}$ 。 利用可操作度椭球体, 在特定位型下能够更加直观、 形象地对机构的灵活性能进行表达。

机构末端速度的可操作椭球体可表达为

$$
\boldsymbol{v}_{p}^{\mathrm{T}}\left(\boldsymbol{J}(\boldsymbol{L}) \boldsymbol{J}^{\mathrm{T}}(\boldsymbol{L})\right)^{-1} \boldsymbol{v}_{p}=1
$$

式中, $v_{p}$ 为末端平台的速度, $\boldsymbol{J}(\boldsymbol{L})$ 为各支链长度为 $\boldsymbol{L}$ 时的雅可比矩阵。

假定需要求解沿着某一方向的可操作度度量, 该方向的单位矢量为 $\boldsymbol{u}_{0}$, 则速度可操作性椭球体的 变化比率为

\begin{tabular}{cccccc}
$\begin{array}{c}\text { 动平台 } x \\
\text { 方向位移 } \\
P_{x} / \mathrm{mm}\end{array}$ & $\begin{array}{c}\text { 动平台 } y \\
\text { 方向位移 } \\
P_{y} / \mathrm{mm}\end{array}$ & $\begin{array}{c}\text { 动平台 } z \\
\text { 方向位移 } \\
P_{z} / \mathrm{mm}\end{array}$ & $\begin{array}{c}\text { 动平台绕 } \\
x \text { 轴转角 } \\
\theta_{x} /\left(^{\circ}\right)\end{array}$ & $\begin{array}{c}\text { 动平台绕 } \\
y \text { 轴转角 } \\
\theta_{y} /\left(^{\circ}\right)\end{array}$ & $\begin{array}{c}\text { 动平台绕 } \\
z \text { 轴转角 } \\
\theta_{z} /\left(^{\circ}\right)\end{array}$ \\
-11.7 & 0.8 & 298.3 & 0.0135 & -0.3129 & -0.1013 \\
-17.5 & 1.6 & 291.8 & -7.4640 & -1.5221 & 48.9189 \\
-21.4 & 1.9 & 288.0 & -8.3264 & -3.2286 & 63.6495 \\
-12.4 & 2.7 & 314.2 & -0.8162 & -0.4665 & -0.2006 \\
-22.3 & -2.299 & 310.4 & 0.2242 & -0.9532 & -30.5569 \\
-36.5 & -3.7 & 305.2 & 1.6016 & -3.3201 & -55.3323 \\
-31.6 & -3.4 & 300.8 & 1.3861 & -2.3363 & -70.3658 \\
-33.7 & -8.5 & 296.6 & 1.6231 & -3.0767 & -87.5943 \\
-34.0 & -12.4 & 291.4 & 3.1562 & -5.2919 & -99.6383 \\
\hline
\end{tabular}

$$
\beta(\boldsymbol{L})=\left(\boldsymbol{u}_{0}^{\mathrm{T}}\left(\boldsymbol{J}(\boldsymbol{L}) \boldsymbol{J}^{\mathrm{T}}(\boldsymbol{L})\right)^{-1} \boldsymbol{u}_{0}\right)^{-1 / 2}
$$

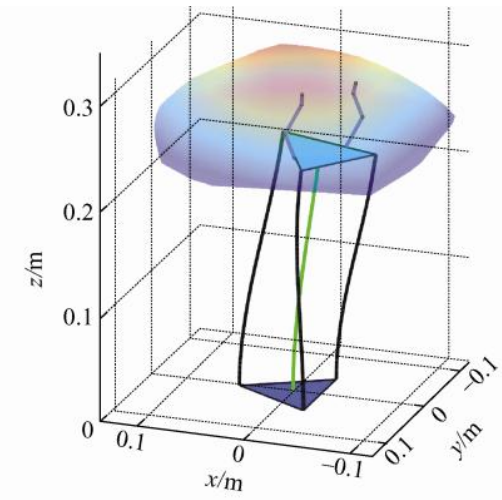

(a) 整体视图

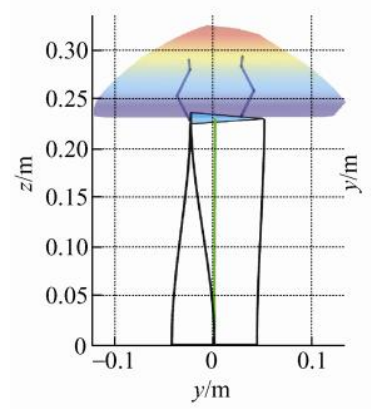

(b) $y-z$ 平面视图

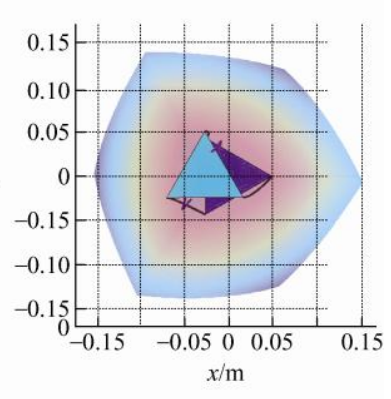

(c) $x-y$ 平面视图

图 8 4-DOF 并联连续体操作手工作空间

通过数值解法可以得到 4-DOF 并联连续体机 构在中性位置 $\left(L_{i}=257 \mathrm{~mm}, i=1,2,3,4\right)$ 时的雅可比

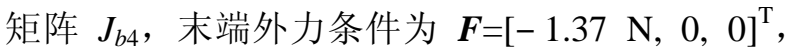
$\boldsymbol{M}=[0,0,0]^{\mathrm{T}}$ 。

$$
\boldsymbol{J}_{b 4}=\left[\begin{array}{cccc}
-0.9 & 1.3 & 1.3 & -0.6 \\
-0.0024 & -0.7707 & 0.7707 & -0.8244 \\
0.3 & 0.1 & 0.1 & -1 \\
0 & 0.1806 & -0.1806 & 0.8201 \\
-0.575 & 0.3143 & 0.3143 & 0.1894 \\
0.0296 & 10.5361 & -10.5389 & -20.1953
\end{array}\right]
$$

通过一个系数因子(Coefficient factor, CF) 将末端 
平台速度的变化转化为位移和角度的变化。 $\beta_{x}, \beta_{y}$ 和 $\beta_{z}$ 分别为动平台沿着 $x, y$ 和 $z$ 轴方向的变化比率, 该参 数能够反映出机构在 $x, y$ 和 $z$ 方向的移动和转动能力。

图 9 为 4-DOF 并联连续体机构在中性位置处位 移可操作椭球体。 $\beta_{x}=1.61, \beta_{y}=0.06$ 和 $\beta_{z}=0.61$, 因此 该机构在 $x$ 方向的移动能力比在 $y, z$ 方向的大, 而 重力方向沿着负 $x$ 轴方向, 所以在相同驱动量的情 况下, 动平台在 $x$ 轴能够产生较大的运动。
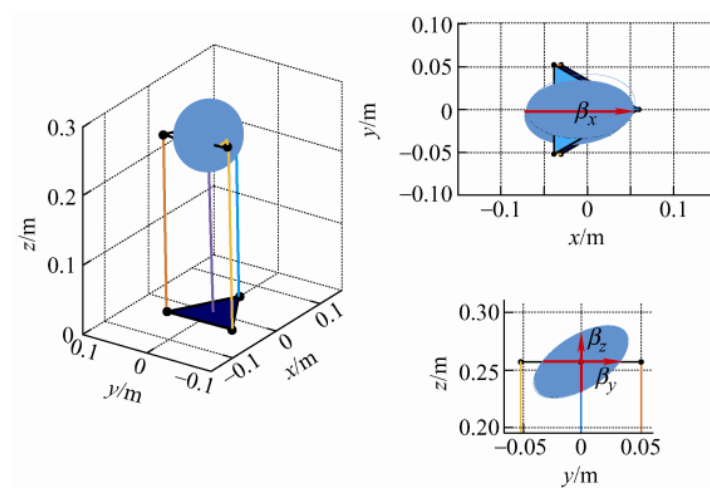

图 9 中性位置操作手位移可操作度椭球体 $(\mathrm{CF}=0.03)$

图 10 为 4-DOF 并联连续体机构在中性位置处 角度操作度椭球体。 $\beta_{x}=0.075, \beta_{y}=0.08$ 和 $\beta_{z}=0.01$, 因此该机构绕着 $x, y$ 轴具有相近的转动能力, 且大 于沿着 $z$ 轴的转动。
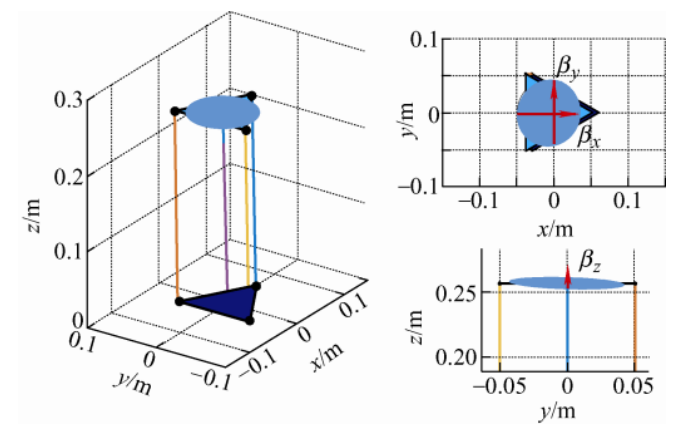

图 10 中性位置操作手角度操作度椭球体 $(\mathrm{CF}=0.06)$

以相同的建模方法得到三支链并联连续体机构 在中性位置的可操作度椭球体, 如图 11 所示, 机构 在 $x$ 轴方向的变化比率大于 $y, z$ 轴方向的, 该结果 与重力沿着负 $x$ 轴方向相一致。在该位型下无论怎 么配置驱动支链都无法产生绕 $z$ 轴的旋转, 因此动 平台在该位型只有外力作用时不具有扭转运动。

进一步分析不具有螺旋副的四支链并联连续体 机构的操作性能, 在 $\boldsymbol{F}=[-1.37 \mathrm{~N}, 0,-1 \mathrm{~N}]^{\mathrm{T}}$, $\boldsymbol{M}=[0,0,0]^{\mathrm{T}}$ 条件下末端平台的位置和角度分别为: $[-1.2 \mathrm{~mm}, 0,256.7 \mathrm{~mm}]^{\mathrm{T}},\left[0,-0.0041^{\circ}, 0\right]^{\mathrm{T}}$ 。此时动 平台仅产生移动和绕 $y$ 轴的转动。

通过对比分析得到所提出的 4-DOF 并联连续 体, 由于驱动末端平台产生扭转运动, 使得操作手 更加的灵活, 并且有效避免连续体机构的奇异位型。
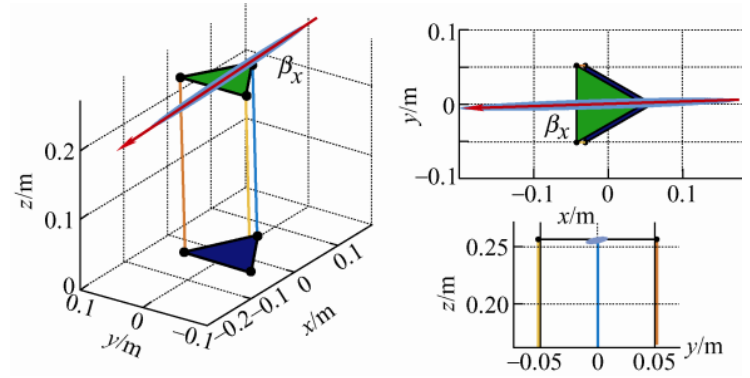

图 11 中性位置操作手位移可操作度椭球体 $(\mathrm{CF}=0.005)$

\section{4 灵活抓取仿真}

将连续体机构与末端抓手相结合, 使得操作手能 够灵活的抓取目标。操作手抓取物体时分为两种抓取 模式，第一种是直接抓取; 第二种灵活抓取。当末端 抓手不需要改变扭转姿态时, 连续体机构可以实现直 接抓取; 当操作手在抓取物体时, 由于其结构的限制, 使得连续体机构必须改变末端抓手的姿态, 此时为第 二种抓取模式，这种模式能够根据物体形状作出相应 的姿态调整, 使末端抓手具有较高的灵活性。

图 12 所示为灵活抓取的实例, 抓取过程分为以 下 4 个阶段。
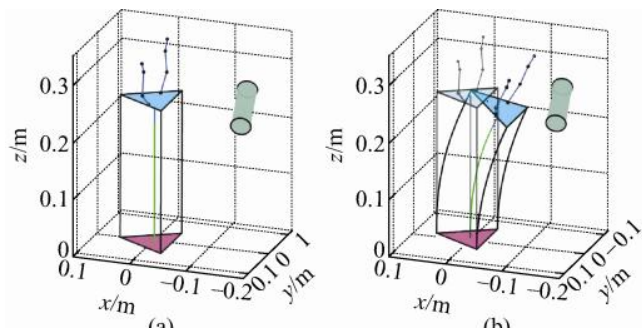

(b)

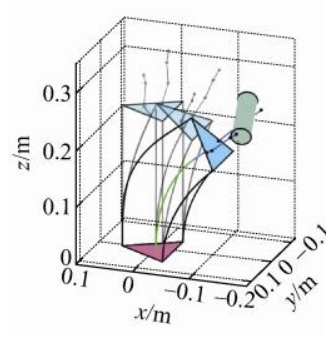

(c)

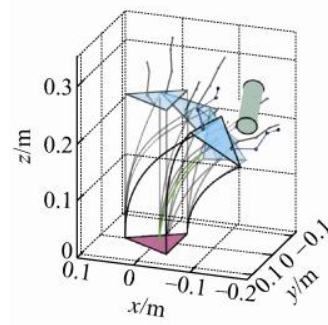

(e)

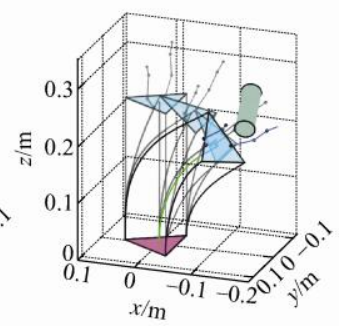

(d)

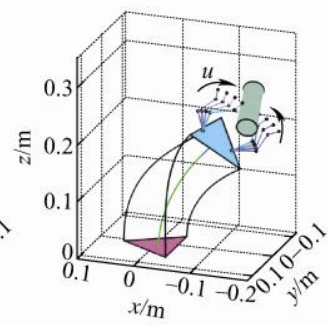

(f)
图 12 灵活抓取物体仿真实例

(1) 操作手处于初始位置, 如图 12a 所示。

(2) 操作手不断弯曲接近目标物体, 见图 $12 \mathrm{~b} 、 12 \mathrm{c}$ 。

（3）接近物体时, 由于末端抓手的结构限制, 目标物体的形状不足以让操作手稳定抓取, 此时连 
续体机构改变末端抓手的姿态以适应物体形状, 如 图 12c $\sim 12 \mathrm{e}$ 所示。

(4) 驱动抓手进行抓取, 完成任务, 如图 $12 \mathrm{f}$ 所示。

\section{4 试验验证}

\section{1 样机设计与试验}

图 13 所示为设计完成的并联连续体操作手样 机, 支链 $1 、 2$ 和 3 同心排列在半径为 $60 \mathrm{~mm}$, 径向 相差 $120^{\circ}$ 的圆上; 支链 4 由内径 $1 \mathrm{~mm}$, 外径 $3 \mathrm{~mm}$ 的空心管构成, 驱动绳通过空心管驱动末端执行器。 末端抓手通过绳驱动实现抓手的闭合抓取, 能够实 现一个自由度的运动, 弹簧提供抓手张开时的弹性 力。将驱动电动机安装在固定座中, 尽可能减少末 端平台所受的外力, 并且提高操作手的轻量化。

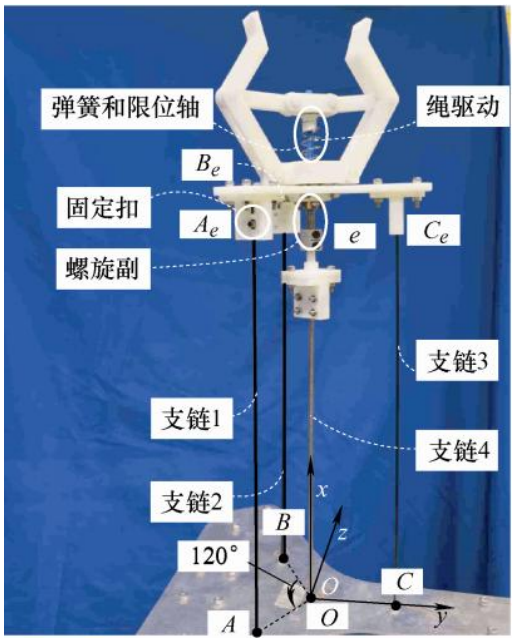

图 13 并联连续体操作手样机

图 14 为并联连续体机构运动分析试验, 试验采 用MostionAnalysis 运动捕捉系统实时记录运动平台 的位置信息。为了避免数据点的丢失, 该试验采用 6 个红外摄像头捕捉。动平台的位置通过安装在上 面的 3 个标记点记录, 基座上的标记点作为定坐标 系 $o-x y z$ 以便于后续的计算。

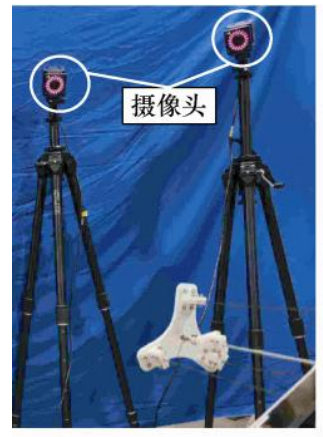

(a)

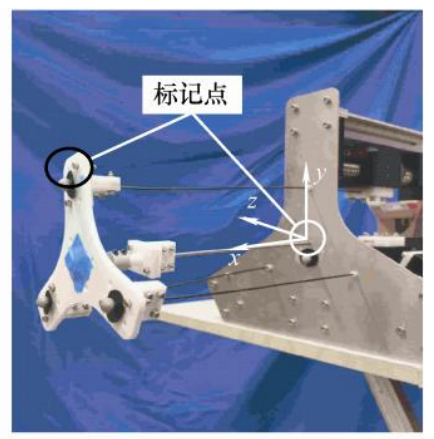

(b)
图 14 MotionAnalysis 试验运动分析

\section{2 自由度验证}

预测求解机构的理论运动模型与样机的实际运 动模型, 得到图 15 中 3 种不同构型下的机构形状,

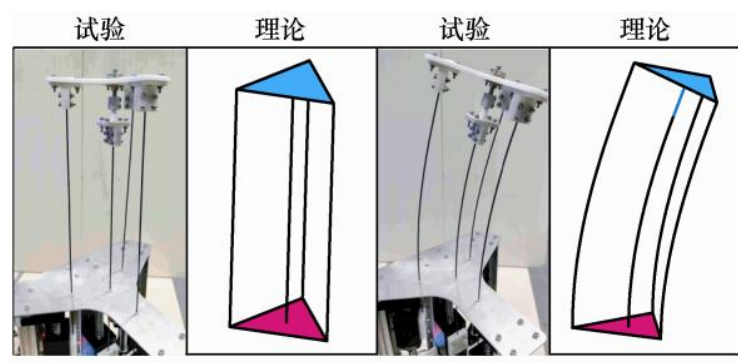

(a) 中性位置

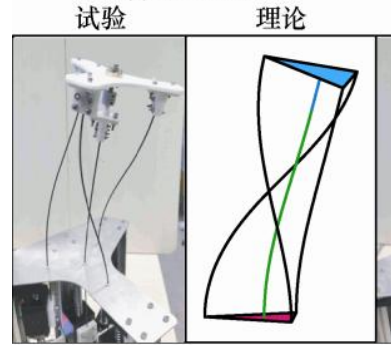

(c) 倾斜扭转 (b) 倾斜
试验

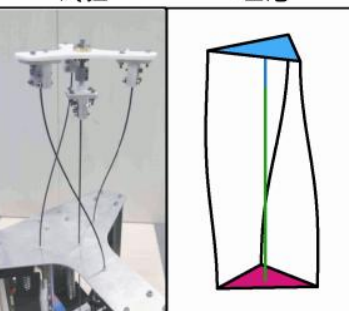

(d) 坚直扭转
图 15 试验构型和理论计算模型

分别为静止状态下的中性位置、绕着 $x, y$ 轴的旋转 位姿, 以及绕着空间中不同轴线的扭转姿态。当所 有连杆长度相等时, 机构处于中性位置; 当等长变 化每条支链的长度时, 动平台会上升或者下降, 实 现沿着 $z$ 轴的移动。当按着特定配置关系同时驱动 支链 $1 、 2 、 3$ 和 4 时, 可以实现操作手绕着 $x, y$ 轴 的旋转。在特定位型条件下，通过驱动柔性支链 4 使得机构获得扭转运动, 从而增加末端抓手的灵活 性。通过对比分析该机构的 3 种运动构型和形状, 证明该机构具有 4 个自由度的运动, 并且理论求解 模型能反映该样机的实际运动。

\section{3 运动学验证}

为了验证运动学模型的正确性, 对表 2 的数值 解进行试验验证, 最终得到了表 3 机构的位置和角 度误差。试验过程中给定各个驱动位型对应的驱动

表 3 4-DOF 并联连续体的运动学验证

\begin{tabular}{cccccc}
\hline $\begin{array}{c}\text { 驱动 } \\
\text { 位型 }\end{array}$ & $\begin{array}{c}\text { 位置误 } \\
\text { 差 } / \mathrm{mm}\end{array}$ & $\begin{array}{c}\text { 百分比 } \\
(\%)\end{array}$ & \multicolumn{3}{c}{ 角度误差 $\left./{ }^{\circ}\right)$} \\
\hline 1 & 2.3006 & 0.94 & -0.1003 & -0.0013 & 0.3503 \\
2 & 1.7932 & 0.73 & 2.51 & 3.7857 & 1.4377 \\
3 & 1.6092 & 0.66 & 1.1126 & 3.8459 & 1.5131 \\
4 & 2.3625 & 0.91 & 0.523 & -1.0507 & 0.21507 \\
5 & 4.8019 & 1.87 & -1.1374 & 0.1149 & -0.2129 \\
6 & 9.4123 & 3.67 & 0.5463 & -0.1151 & -1.0733 \\
7 & 0.0117 & 2.62 & 1.5319 & 0.8321 & 1.3461 \\
8 & 7.3547 & 2.89 & -1.4608 & 1.0381 & 2.5639 \\
9 & 6.7406 & 2.67 & -0.5761 & 0.0863 & 3.0073 \\
\hline
\end{tabular}


量, 测量末端平台的三维坐标, 分析各驱动位型的 误差。其中位置误差为空间三维坐标的总误差绝对 值, 而误差百分比为位置误差绝对值与支链总长度 平均值的比值。根据坐标可以解算出动平台旋转的 角度, 角度误差分别为绕 $x, y, z$ 的角度误差。

表 3 中机构位置的最大误差不超过 $10 \mathrm{~mm}$, 误 差百分比不超过 $4 \%$, 三个旋转角度误差不超过 $4^{\circ}$, 由于样机装配误差和各支链弧长测量误差的存在, 文中所构建的 4-DOF 并联连续体运动学模型能够较 为准确反映该机构的运动特性, 从侧面反映所构建 模型的正确性。

\section{4 物体抓取试验}

图 16 18 为操作手两种抓取模式的物体抓取 试验, 分别为 1-DOF 的直接抓取、2-DOF 直接抓取 和灵活抓取。图中坐标系建立在基座中, 与图 13 的坐标系相一致, 连续体绕着 $x$ 轴旋转实现 1-DOF 的弯曲, 绕着 $x, y$ 旋转实现 2-DOF 的弯曲。从图中 可以看出直接抓取分为初始位置、靠近和抓取三个 过程, 而灵活抓取分为初始位置、靠近、适应和抓 取四个过程。

图 16 给出了三组物体抓取试验, 分别对应图 16 中(1) (3)、(4) (6)和(7) (9), 物体位于 $x-z$ 平面 内, 并且物体的周向尺寸在抓手的抓取范围内。从 位置和姿态上来看, 连续体只需要实现绕 $x$ 轴的弯 曲, 并且驱动操作手不断靠近物体, 最后成功抓取 目标。

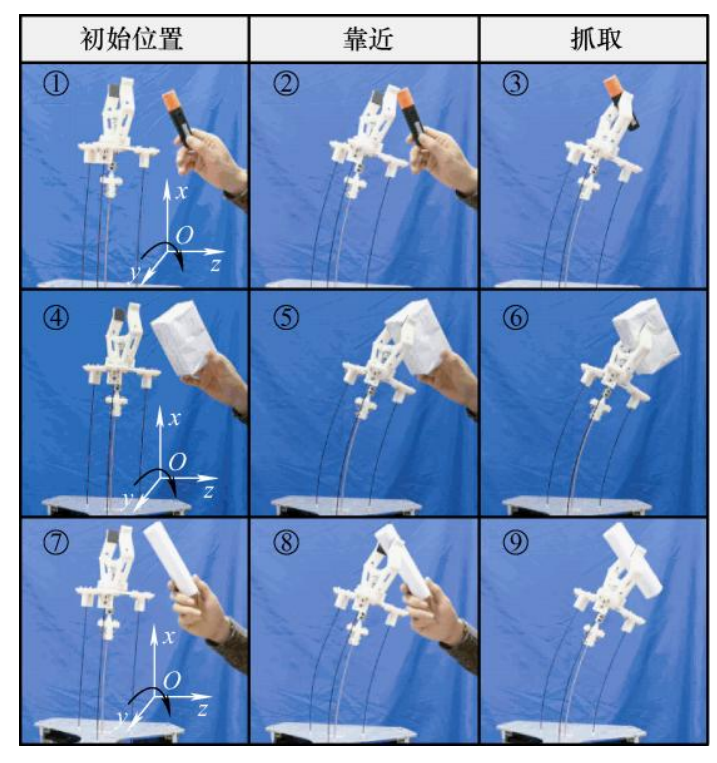

图 16 直接抓取(1-DOF 弯曲)

图 17 分别给出了(1)〜 (3)、(4)～(6)和(7)～(9)三组 物体试验, 物体位于距 $x-z$ 平面一定距离且平行于 该平面的平面内, 操作手不能通过一个弯曲自由度 的运动实现抓取任务, 因此连续体绕 $x, y$ 轴旋转实
现 2 自由度弯曲运动。

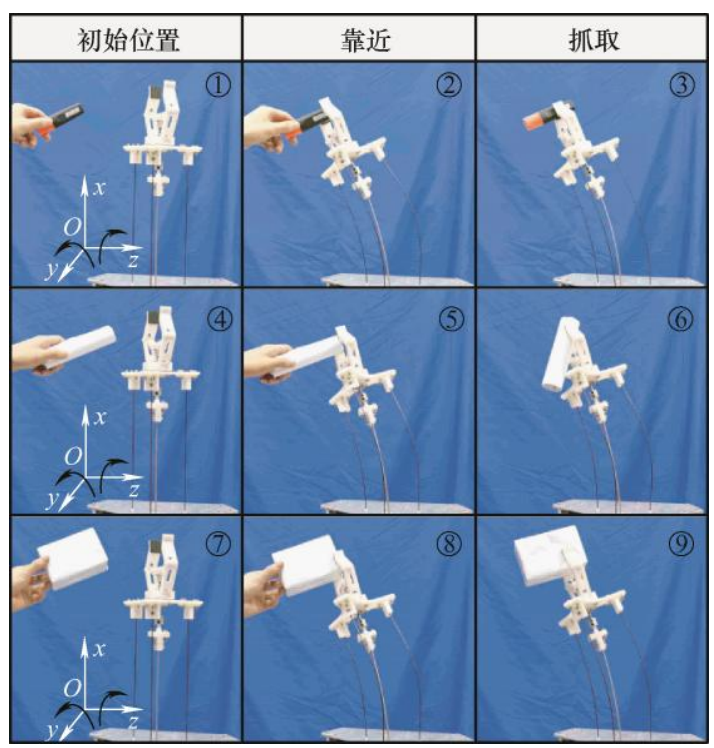

图 17 直接抓取(2-DOF 弯曲)

以上这两种抓取运动是操作手的直接抓取模 式, 物体的周向尺寸和姿态使得抓手能直接抓取, 不需要提供操纵手的扭转运动, 从试验结果也可以 反映出该机构未发生扭转。

图 18 给出了操作手三组灵活抓取物体的实 例, 分别为图 18 中(1) (4)、(5)〜 (8)和 (9)〜 (12), 图 18(1) (4)验证了图 12 给出的一组灵活抓取的 仿真实例。该操作手对不同形状的物体, 能够灵 活抓取, 初始时刻操作手保持一定的姿态和位 置, 在靠近物体的过程中, 连续体不断弯曲运动, 当发现末端抓手姿态不能成功抓取物体时, 该机 构会提供一个扭转自由度, 使得操作手灵活的适 应物体形状, 完成抓取。

\section{5 结论}

（1）本文设计了一种新型柔性四支链并联连续 体机构, 通过将该机构与末端绳驱动的抓手相结合, 制作完成具有灵活抓取性能的柔性操作手。

(2) 通过等效柔性支链和螺旋理论的方法分析 得到该柔性连续体机构的自由度为 4 , 并且通过试 验验证了该机构自由度。

(3) 采用经典的 Cosserat Rod 模型对 4-DOF 并 联连续体机构进行运动学建模, 并且通过试验验证 了所建模型的正确性, 为运动学控制提供理论基础。

(4) 4-DOF 并联连续体机构能够提供一个额外的 扭转自由度, 通过仿真实例和试验验证该机构具有更 好的操作性能, 能够实现操作手更加柔顺和灵活的 抓取。 


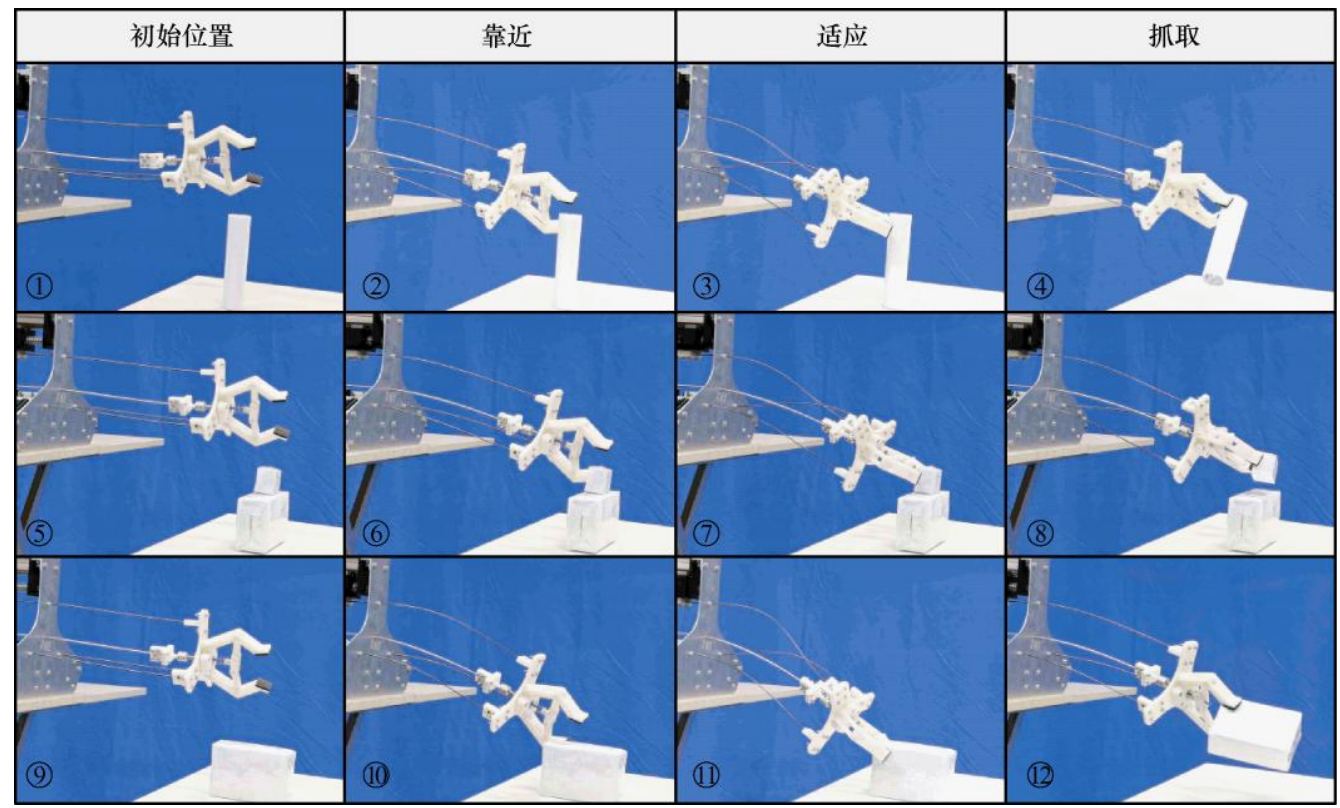

图 18 灵活抓取物体

\section{参 考 文 献}

[1] WEBSTER R J, JONES B A. Design and kinematic modeling of constant curvature continuum robots: A review $[\mathrm{J}]$. The International Journal of Robotics Research, 2010, 29(13): 1661-1683.

[2] BRYSON C E, RUCKER D C. Toward parallel continuum manipulators[C]// 2014 IEEE International Conference on Robotics and Automation (ICRA). IEEE, 2014: 778-785.

[3] 于靖军, 郝广波, 陈贵敏, 等. 柔性机构及其应用研究 进展 $[\mathrm{J}]$. 机械工程学报，2015，51(13)：53-68.

YU Jingjun, HAO Guangbo, CHEN Guimin, et al. State-of-art of compliant mechanisms and their applications $[\mathrm{J}]$. Journal of Mechanical Engineering. 2015, 51(13): 53-68.

[4] SIMAAN N, TAYLOR R, FLINT P, et al. A dexterous system for laryngeal surgery[C]// Proceeding of 2004 IEEE International Conference on Robotics and Automation, 2004: 351-357.

[5] 徐凯, 刘欢. 多杆连续体机构: 构型与应用 [J]. 机械工 程学报, 2018, 54(13): 25-33.

XU Kai , LIU Huan. Multi-backbone continuum mechanisms : Forms and applications[J]. Journal of Mechanical Engineering， 2018， 54(13): 25-33.

[6] ZHAO J R, ZHENG X D, ZHENG M H, et al. An endoscopic continuum testbed for finalizing system characteristics of a surgical robot for NOTES procedures $[\mathrm{C}] / / 2013$ IEEE/ASME International Conference on Advanced Intelligent Mechatronics. IEEE, 2013: 63-70.

[7] XU K, ZHAO J R, FU M X. Development of the SJTU unfoldable robotic system (SURS) for single port laparoscopy[J]. IEEE/ASME Transactions on Mechatronics, 2014, 20(5): 2133-2145.

[8] LIU S T, YANG Z X, ZHU Z J, et al. Development of a dexterous continuum manipulator for exploration and inspection in confined spaces[J]. Industrial Robot: An International Journal，2016， 43(3): 284-295.

[9] XU K, ZHAO J R, QIU D, et al. A pilot study of a continuum shoulder exoskeleton for anatomy adaptive assistances[J]. Journal of Mechanisms and Robotics, 2014, 6(4): 041011.

[10] XU K, WANG Y, YANG Z X. Design and preliminary experimentation of a continuum exoskeleton for self-provided bilateral rehabilitation[C]//2014 IEEE International Conference on Information and Automation (ICIA). IEEE, 2014: 327-332.

[11] LIU H, ZHANG Z Y, DONG T L, et al. A single-actuator gripper with a working mode switching mechanism for grasping and rolling manipulation[C]// 2018 IEEE/ASME International Conference on Advanced Intelligent Mechatronics (AIM). IEEE, 2018: 359-364.

[12] XU K, LIU H, ZHANG Z, et al. Wrist-powered partial hand prosthesis using a continuum whiffle tree mechanism: A case study[J]. IEEE Transactions on 
Neural Systems and Rehabilitation Engineering, 2018, 26(3): 609-618.

[13] YANG Z, ZHU X, XU K. Continuum Delta Robot: a Novel Translational Parallel Robot with Continuum Joints[C]// 2018 IEEE/ASME International Conference on Advanced Intelligent Mechatronics (AIM). IEEE, 2018: 748-755.

[14] FESTO. Bionic Tripod 3.0. [EB/OL]. https : //www.festo.com/group/en/cms/10240.htm, 2011.

[15] TILL J, ALOI V, RUCKER C. Real-time dynamics of soft and continuum robots based on Cosserat rod models[J]. The International Journal of Robotics Research, 2019, 38(6): 723-746.

[16] OREKHOV A L, BRYSON C E, TILL J, et al. A surgical parallel continuum manipulator with a cable-driven grasper[C]// 201537 th Annual International Conference of the IEEE Engineering in Medicine and Biology Society (EMBC). IEEE， 2015: 5264-5267.

[17] YOUNG E M, KUCHENBECKER K J, GERLING G. Design of a parallel continuum manipulator for 6-DoF fingertip haptic display[C]// 2017 IEEE World Haptics Conference (WHC). IEEE, 2017: 599-604.

[18] YOUNG E M, KUCHENBECKER K J. Implementation of a 6-DOF parallel continuum manipulator for delivering fingertip tactile cues[J]. IEEE Transactions on Haptics, 2019, 12(3): 295-306

[19] 方跃法, 林华杰. 连续体并联抓取机器人的结构设计及 运动学分析 $[\mathrm{J}]$. 北京交通大学学报, 2019, 43(4): 80 .
FANG Yuefa, LIN Huajie. Structural designand kinematics analysis of the continuum parallel grasping manipulator[J]. Journal of Beijing Jiaotong University, 2019, 43(4): 80 .

[20] WU G L, SHI G L. Experimental statics calibration of a multi-constraint parallel continuum robot[J]. Mechanism and Machine Theory, 2019, 136: 72-85.

[21] 陈子明, 张扬, 黄坤, 等. 一种无伴随运动的对称两转 一移并联机构 [J]. 机械工程学报，2016，52(3): 9-17. CHEN Ziming, ZHANG Yang, HUANG Kun, et al. Symmetrical 2R1T parallel mechanism without parasitic motion[J]. Journal of Mechanical Engineering, 2016, 52(3): 9-17.

[22] JONES B A, GRAY R L, TURLAPATI K. Three dimensional statics for continuum robotics[C]// 2009 IEEE/RSJ International Conference on Intelligent Robots and Systems. IEEE， 2009: 2659-2664.

[23] ANTMAN S S, MARSDEN J E, SIROVICH L. Nonlinear problems of elasticity[M]. 2nd ed. New York: Springer, 2005

[24] YOSHIKAWA T. Manipulability of robotic mechanisms [J]. The international journal of Robotics Research, 1985, 4(2): 3-9.

作者简介: 汪培义, 男, 1995 年出生, 博士研究生。主要研究方向为机 器人机构学和连续体机器人。

E-mail: 19116019@bjtu.edu.cn 郭盛(通信作者), 男, 1972 年出生, 博士, 教授, 博士研究生导师。主 要研究方向为机器人机构学。

E-mail: shguo@bjtu.edu.cn 